\title{
Closure to "A cantilever approach to estimate bending stiffness of buildings affected by tunnelling" by Twana K. Haji, Alec M. Marshall, and Walid Tizani
}

\author{
Twana Kamal Haji ${ }^{a, *}$, Alec M. Marshall ${ }^{\mathrm{a}}$, Walid Tizani ${ }^{\mathrm{a}}$ \\ ${ }^{a}$ Department of Civil Engineering, Faculty of Engineering, University of Nottingham, \\ Nottingham, United Kingdom.
}

The discussion of Franza and DeJong (2018) raises some interesting points. The discussion covers three main areas, namely [1] the proposed method for estimating building bending stiffness, [2] the assumed boundary condition for the building base columns, and [3] the utilisation of the proposed method. Below are some additional comments organised according to these points.

[1] Franza and DeJong (2018) highlighted that the point-load analogy (i.e. bending stiffness $=$ force/deflection) used in Haji et al. (2018) to develop equations for estimating the bending stiffness of a building includes the effect of shear and bending deformations, and referred to this as the 'total stiffness'. It was suggested that the contribution of shear and bending should be distinguished for the evaluation of building bending stiffness, which is sensible. We simply note that the intention of the proposed method was not to follow a strict analytical scheme; it is intended as a simplified approach to estimate building bending stiffness that attains a good level of accuracy (by virtue of its development with rigorous numerical analyses) and is able

\footnotetext{
*Corresponding author

Email address: twana.k.haji@gmail.com (Twana Kamal Haji )
} 
to capture features that are not incorporated in existing analytical methods (e.g. constraint of building due to length in unaffected zone).

Franza and DeJong (2018) also commented on the comparison of total bending stiffness, $K_{b}$, with values provided using the method of Franzius et al. (2006), where flexural rigidity $(E I)$ does not account for shear-type flexibility. We agree that, due to the underlying differences in the approaches, a strict comparison between the methods is not possible, however we felt that an attempt to put results within the context of existing methods was worthwhile. The original paper discusses some differences between the proposed method and those provided by Franzius et al. (2006) and Potts and Addenbrooke (1997), including boundary conditions and length of building affected by tunnelling; addition of 'total' versus 'bending only' stiffness to the list of differences betwen the methods is a useful contribution.

[2] Franza and DeJong (2018) noted that the physical basis of the assumed fixed boundary conditions in the original paper should be clarified. We completely agree that the role of the foundation scheme is an important parameter in determining the response of the building. The assumed fixed boundary condition at the base of columns may not be realistic for some foundation types, such as single footings or combined (strip) footings running parallel to the tunnel axis, since horizontal displacements and rotations can have an impact on the building behaviour. For this reason, the methodology is most applicable to reasonably large, reinforced concrete framed buildings which are likely to have combined or raft foundations rather than single shallow footings. In such foundation cases, reinforced concrete base columns would behave reasonably rigidly, in a way that is close to a fixed support. We 
would also note that a fixed boundary is a popular option in the structural analysis and design of reinforced concrete buildings in static cases when columns are subjected to large axial forces and small bending moments (due to lateral loads), where large foundations are provided (Duggal, 2009).

[3] Franza and DeJong (2018) presented two main points related to the utilisation of the proposed methodology. First, it was noted that the length of the building influenced by tunnelling is fixed and does not depend on soil-structure interaction. The developed equations will of course lead to more realistic results when the building length affected by tunnelling is predicted accurately, however the focus of the proposed method was not to concentrate on this aspect. In addition, results from the proposed method show that building stiffness does not vary considerably if two or more building panels are affected (refer to Figure 15a of the original paper), which will be the case for most practical scenarios).

Second, Franza and DeJong (2018) commented on the applicability of the proposed method within the currently accepted modification factor frameworks (e.g. works proposed by Franzius et al. (2006) and Giardina et al. (2015)). These frameworks mainly depend on the flexural rigidity (EI), while the method proposed by Haji et al. (2018) considers important additional parameters that influence the bending stiffness of a building. As previously discussed, this makes comparison of results from the proposed methodology against those from existing methods difficult. We feel that development of building damage assessment methods that incorporate some of the important features addressed by Haji et al. (2018) are needed but agree with Franza and DeJong (2018) in that this is an area that requires further work. 
Duggal, S., 2009. Design of steel structures, 3rd Edition. Tata McGraw-Hill Education.

Franzius, J. N., Potts, D. M., Burland, J. B., 2006. The response of surface structures to tunnel construction. Proceedings of the Institution of Civil Engineers-Geotechnical Engineering 159 (1), 3-17.

Giardina, G., DeJong, M. J., Mair, R. J., 2015. Interaction between surface structures and tunnelling in sand: Centrifuge and computational modelling. Tunnelling and Underground Space Technology 50, 465-478.

Haji, T. K., Marshall, A. M., Tizani, W., 2018. A cantilever approach to estimate bending stiffness of buildings affected by tunnelling. Tunnelling and Underground Space Technology 71, 47-61.

Potts, D., Addenbrooke, T., 1997. A structure's influence on tunnellinginduced ground movements. Proceedings of the ICE-Geotechnical Engineering 125 (2), 109-125. 